\title{
Singular solutions to Maxwell's equations and their significance for geomagnetic induction
}

\author{
Detlef Wolf Department of Physics, University of Toronto, Toronto, \\ Ontario MSS 1A7, Canada
}

Received 1983 March 11 ; in original form 1982 December 29

\section{Introduction}

In geomagnetism the interpretation of induction anomalies has frequently encountered difficulties. Sometimes the problems may be overcome if channelled currents are taken into account. This idea was introduced by Japanese workers to explain the characteristics of the Central Japan anomaly in a consistent manner (Rikitake 1959). But whereas their channelling models showed some resemblance to electrical circuits, later work has usually considered the three-dimensionality of the problem. Dyck \& Garland (1969) correctly pointed out that current channelling would always be present unless: (1) the source fields are local or (2) the conductivity of the host rocks is vanishing. For the real Earth neither condition applies rigorously.

Any serious interpretation of induction anomalies must consider their frequency response. If the frequency dependence is small and phase shifts of the magnetic field across the anomaly are negligible, either (1) local induction is predominant and close to the inductive limit or (2) local induction is subordinate and the response caused by channelled currents. For moderate frequencies the former explanation requires high conductivities or conductors of large extent. In contrast to this, the applicability of the channelling concept is more general and not confined to any frequency range.

In a critical review of acceptable mechanisms leading to a frequency-independent response, Summers (1982) has discussed the possibility of an alternative local model. Its behaviour must be characterized by anomalous horizontal fields $H_{y \mathrm{a}}$ that are large com. pared to the corresponding normal part $H_{y \mathrm{n}}$. The total horizontal field $H_{y}$ is therefore essentially anomalous. For uniform inducing fields the normal vertical field vanishes and $H_{z}$ is completely anomalous. Then, if $H_{z}$ were of the same order as $H_{y \mathrm{a}}$ over a broad range of frequencies, the total field ratio $H_{z} / H_{y}$ would be approximately frequency-independent in that range. With the (incorrect but conventional) assumption that $H_{y \mathrm{a}}<H_{y \mathrm{n}}$, the constancy of $H_{z} / H_{y \mathrm{n}}$ could then be inferred and the local response erroneously be classified as frequency-independent.

In an attempt to demonstrate the physical reasonableness of his hypothesis, Summers (1982) numerically calculated the magnetic field at a particular point above but slightly displaced from the centre of a locally bounded two-dimensional conductor for a large range of frequencies (see his figs 2,3 and 4). The response curves show that, with decreas- 
ing frequency $\omega$, the ratios $H_{z} / H_{y \mathrm{a}}$ and $H_{z} / H_{y}$ reach the same constant value, whereas the normalized ratio $H_{z} / H_{y_{n}}$ increases sharply, such that

$\lim H_{z} / H_{y \mathrm{n}}=\infty$

$\omega \rightarrow 0$

is implied

The correctness of Summers' concept of frequency-independent local induction is obviously closely related to the correctness of this low-frequency behaviour of the anomalous field. Regardless of that, the strong increase of $H_{y \mathrm{a}}$ and $H_{z}$ with period is certainly remarkable in itself, particularly because Summers' observation point is well removed from all strong conductivity contrasts. In an attempt to provide an independent check of this feature, the low-frequency limit of the response of several model conductors is discussed here. The general conclusion will be that, for configurations adequate to the Earth, the solutions are incompatible with excessively large anomalous fields at points sufficiently removed from conductivity contrasts.

\section{Singularities of the magnetic field}

The behaviour of the magnetic field in the vicinity of sharp conductivity contrasts is known from analytical solutions. Raval, Weaver \& Dawson (1981) have solved the $E$-polarization case for a homogeneous half-space overlain by a perfectly conducting half-plane and subject to uniform inducing fields. Their solution involves a single characteristic length, i.e. the inductive skin depth $\delta$ of the half-space for uniform fields. If all lengths are scaled with respect to this inductive length, the system's response is unique, i.e. independent of frequency. Raval et al. (1981) show that in the vicinity of the edge of the perfectly conducting half-plane $H_{y \mathrm{a}}$ and $H_{z}$ are both of the order $(r / \delta)^{-1 / 2}$, where $r$ denotes the distance from the edge. But for $\omega \rightarrow 0$ we have $\delta \rightarrow \infty$. Thus, if the observation point is fixed at $r$, both $H_{y \mathrm{a}}$ and $H_{z}$ tend to infinity. Summers (1982) has referred to this low.frequency limit as 'electrically close'.

For the real Earth, however, the significance of this result is only limited. There are at least two reasons for that.

(1) Any configuration of conductors and source fields adequate to the Earth has also geometrically defined characteristic lengths. In the previous example this could possibly be realized by introducing a second half-plane to the top of the uniform half-space. If the distance between the edges of the perfectly conducting two half-planes is denoted by $2 L$, the geometry of the response, normalized with respect to $\delta$, is not frequency-independent since it depends on $L / \delta$. This, however, renders the concept of 'electrical closeness' inadequate. As demonstrated below, it also implies that, for $\omega \rightarrow 0$, the magnetic field remains finite except at the edge points themselves.

(2) With decreasing frequency, the model of perfectly conducting sheets becomes poor. For very low frequencies, any conductor effectively has infinite skin depth, and induction ceases to be important.

In the following, we will discuss the significance of (1) in terms of a heuristic model. In it the uniform half-space underlying the two half-planes is replaced by a perfectly conducting whole-plane, here denoted as the conductopause. Its depth $\delta$ below the surface simulates the skin depth of the half-space and is therefore considered as frequency-dependent. Correspondingly, for high frequencies, $\delta$ will be small, whereas for $\omega \rightarrow 0$ it increases beyond all limits. Fig. 1 shows the general geometry of this configuration. The solution for the magnetic field may be obtained by conformal mapping methods. For a uniform field this has been outlined by Wolf (1983). Here we confine ourselves to the vertical component of 


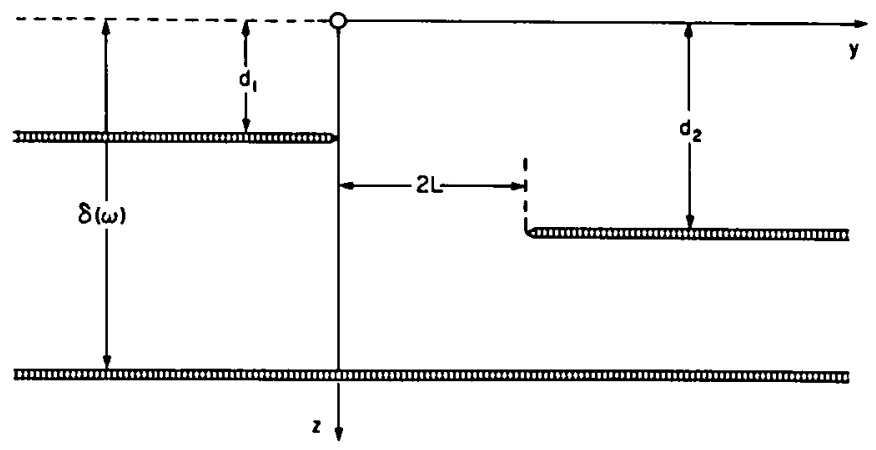

Figure 1. General conductor configuration. The depths of the conductors below the surface $z=0$ are $d_{1}$ (left half-plane), $d_{2}$ (right half-plane) and $\delta$ (whole-plane), which is frequency-dependent. In Figs 2 and $3 d_{1}=d_{2}=0$ is assumed.

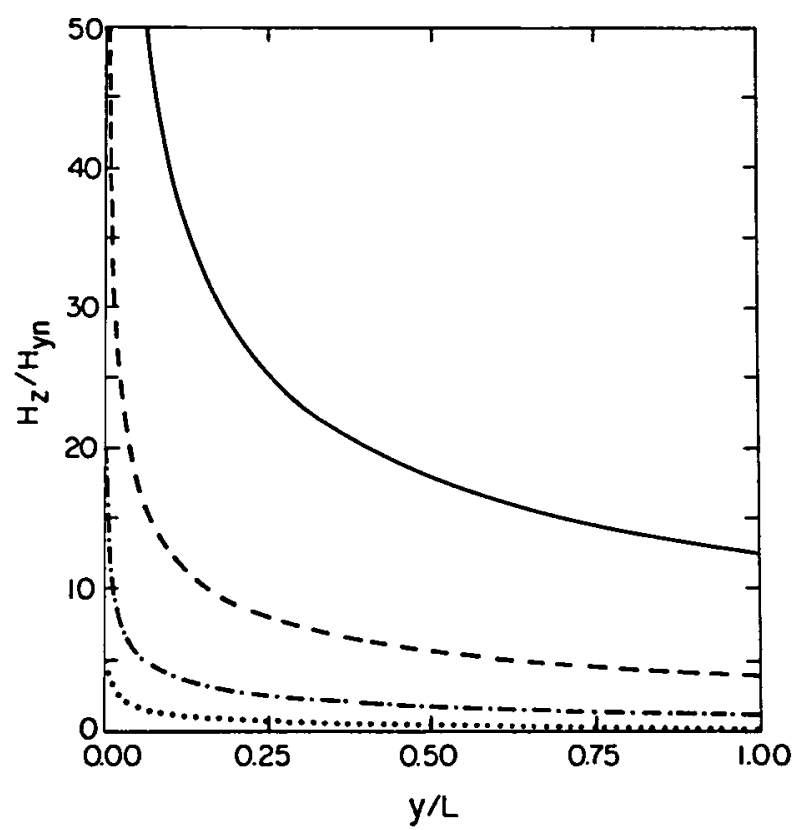

Figure 2. Response characteristics of normalized field ratio $H_{2} / H_{v n}$ for $\delta / L=1$ (dotted), $\delta / L=10$ (dotdashed), $\delta / L=100$ (dashed) and $\delta / L=1000$ (solid). The right half-plane is excluded from the model. The profile is at $z=0$.

the magnetic field and consider the symmetrical case $d_{1}=d_{2}=0$. This situation has already been discussed by Schmucker (1970).

If the right half-plane is excluded from the model for a moment, the configuration is very simple. Fig. 2 shows the associated normalized response $H_{z} / H_{y n}$ for different depths of the conductopause. As pointed out by Siebert (1965), $\delta$ is the only characteristic length inherent in this model. An increase in $\delta$ by a factor of 10 , say, therefore extends the edge effect by the same factor. If in particular $\delta \rightarrow \infty$, i.e. $\omega \rightarrow 0$, the conductopause moves to infinity. Then every point is effectively at the edge of the half-plane, and the anomalous field becomes infinite everywhere. 


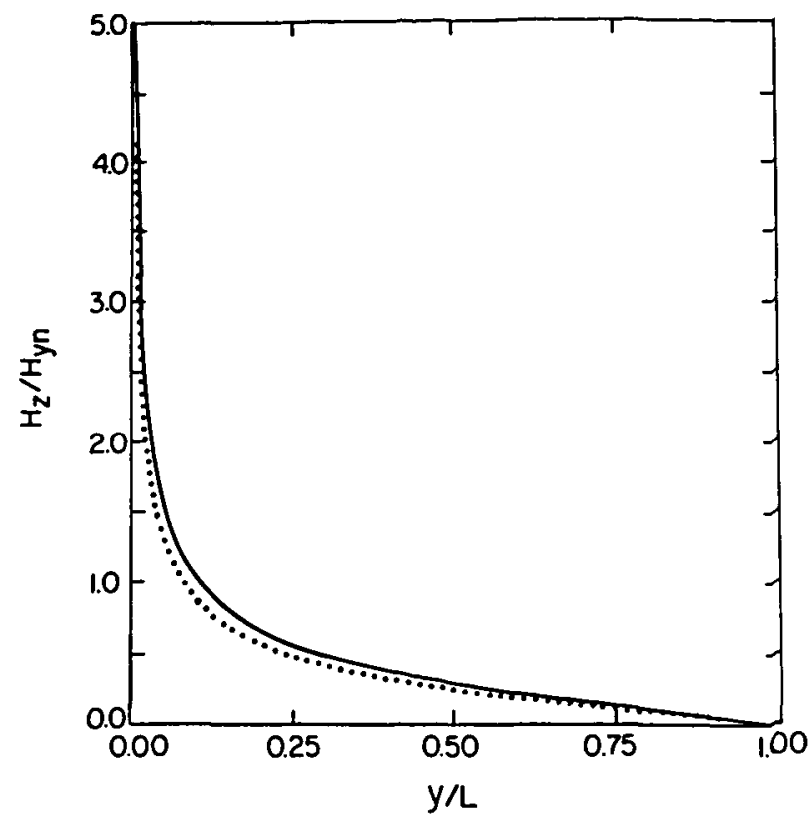

Figure 3. Response characteristics of normalized field ratio $H_{z} / H_{y \mathrm{n}}$ for $\delta / L=1$ (dotted) and $\delta / L>10$ (solid). Both half-planes are included in the model. The profile is at $z=0$.

This result is only of limited use for us and related to the indeterminacy of induction in a single half-plane by a uniform field. However, if the second half-plane is incorporated into the model, the singular character of the low-frequency limit is removed. Then the distance $2 L$ between the two edge points represents a frequency-independent characteristic length. Fig. 3 again shows the ratio $H_{z} / H_{y n}$ for different values of $\delta$. This time the solution remains bounded at all but the edge points even for $\delta \rightarrow \infty$. But since this is equivalent to $\omega \rightarrow 0$ in our model, the situation resembles the low-frequency limit considered by Summers (1982).

The controversial point now becomes obvious. Even though Summers' model does have frequency-independent characteristic lengths, its anomalous field grows very large for low frequencies. Such a behaviour, however, is in clear contradiction to the results of the present study. It is also not confirmed by the conformal mapping solution for a half-cylindrical bulge projecting from a perfectly conducting half-space (Schmucker et al. 1966; Greenhouse, Parker \& White 1973). This idealized configuration is closely related to the model discussed by Summers (1982). Notably the associated magnetic field is non-singular everywhere. $\bar{A}$ consideration of corresponding finitely conducting two-dimensional bodies, however, should lead all the more to continuous solutions for the magnetic field. Parker (1968) solved the problem of induction, by a normally incident field, in a finitely conducting thin strip analytically. Here the magnetic field has logarithmic singularities at the edges, which are of lower order than the $(r / \delta)^{-1 / 2}$ singularity associated with the corresponding perfectly conducting configuration (Abramowitz \& Stegun 1965, p. 68). As expected, the region of strong anomalous fields becomes increasingly confined for decreasing frequency in Parker's solution. Kertz (1960) derived analytical solutions for induction in a homogeneous cylindrical body of finite conductivity by a transverse magnetic field. The magnetic field remains bounded everywhere and particularly at the surface of the cylinder. 


\section{Conclusions}

In conclusion we may state that the correctness of Summers' (1982) response curves (see his figs 3 and 4) must be questioned. The main reason for this is that a dramatic increase of the anomalous field for decreasing frequency, i.e. towards the resistive limit is required. Intuitively, such response characteristics appear highly unusual. As demonstrated by the present discussion, its theoretical justification is likewise difficult. It is therefore suggested that Summers' numerical results be checked against analytical solutions. Kertz's (1960) cylindrical body seems very appropriate for this and also resembles the configuration discussed by Summers (1982) very closely. As the cylinder is embedded into a nonconducting medium, spurious channelling effects are excluded and induction is strictly local. Consequently, Kertz's response curves are as expected, i.e. frequency independence is confined to the high-frequency limit. An easy test of the accuracy of Summers' numerical code is therefore possible, and the character and frequency range of the discrepancies between the two solutions can be determined.

\section{Acknowledgment}

I would like to thank Dr P. Weidelt for drawing my attention to the conformal mapping solution for the half-cylindrical bulge.

\section{References}

Abramowitz, M. \& Stegun, I. A., 1965. Handbook of Mathematical Functions, Dover, New York.

Dyck, A. V. \& Garland, G. D., 1969. A conductivity model for certain features of the Alert anomaly in geomagnetic variations, Can. J. Earth Sci., 6, 513-516.

Greenhouse, J. P., Parker, R. L. \& White, A., 1973. Modelling geomagnetic variations in or near an ocean using a generalized image technique, Geophys. J. R. astr. Soc., 32, 325-338.

Kertz, W., 1960. Leitungsfaehiger Zylinder im transversalen magnetischen Wechselfeld, Beitr. Geophys., $69,4-28$.

Parker, R. L., 1968. Electromagnetic induction in a thin strip, Geophys. J. R. astr. Soc., 14, 487-495.

Raval, U., Weaver, J. T. \& Dawson, T. W., 1981. The ocean-coast effect re-examined, Geophys. J. R. astr. Soc., 67, 115-123.

Rikitake, T., 1959. Anomaly of geomagnetic variations in Japan, Geophys. J. R. astr. Soc., 2, 276-287.

Schmucker, U., 1970. Anomalies of geomagnetic variations in the south-western United States, Bull. Scripps Inst. Oceanogr., 13, 1-165.

Schmucker, U., Forbush, S. E., Hartmann, O., Giesecke, A. A. (Jr), Casaverde, M., Castillo, J., Salgueiro, R. \& del Pozo, S., 1966. Electrical conductivity anomaly under the Andes, Yb. Carnegie Instn Wash., 65, 11-28.

Siebert, M., 1965. Bemerkungen zur Untersuchung der Norddeutschen Leitfaehigkeitsanomalie, in Proc. Symp. 'Erdmagnetische Tiefensondierung', Goslar, pp. 108-129. University of Braunschweig.

Summers, D. M., 1982. On the frequency response of induction anomalies, Geophys. J. R. astr. Soc., 70, 487-502.

Wolf, D., 1983. Inductive coupling between idealized conductors and its significance for the geomagnetic coast effect, J. Geophys., 52, 22-33. 